\title{
Decreased plasma decorin levels following acute ischemic stroke: Correlation with MMP-2 and differential expression in TOAST subtypes
}

\author{
YUAN-ZHI XU*, KAI-JUN ZHAO*, ZHI-GANG YANG, YU-HUI ZHANG, YONG-WEI ZHANG, \\ BO HONG and JIAN-MIN LIU
}

Department of Neurosurgery, Changhai Hospital, Second Military Medical University, Shanghai 200433, P.R. China

Received March 30, 2012; Accepted August 1, 2012

DOI: $10.3892 / \mathrm{mmr} .2012 .1108$

\begin{abstract}
Accumulating evidence suggests that extracellular matrix (ECM) remodeling plays a significant role following acute ischemic stroke (AIS). Decorin (DCN) is a well-recognized molecule present in the ECM; however, the role of DCN in AIS remains unknown. The present study aimed to investigate whether plasma concentrations of DCN are altered in patients following an AIS and whether they are correlated with matrix
\end{abstract}

Correspondence to: Professor Bo Hong or Professor Jian-Min Liu, Department of Neurosurgery, Changhai Hospital, Second Military Medical University, 168 Changhai Road, Shanghai 200433, P.R. China

E-mail: hongbosmmu@yeah.net

E-mail: liu118@vip.163.com

*Contributed equally

Abbreviations: AF, atrial fibrillation; AIS, acute ischemic stroke; ANOVA, analysis of variance; APTT, activated partial thromboplastin time; AUC, area under the curve; CEI, cardioembolic infarcts; CT, computed tomography; DBP, diastolic blood pressure; DM, diabetes mellitus; ECM, extracellular matrix; EGF-R, epidermal growth factor; ELISA, enzyme-linked immunosorbent assay; FDP, fibrinogen degradation products; FIB, fibrinogen; GAG, glycosaminoglycan; HDL, high-density lipoprotein; IGF-1R, insulin-like growth factor-1 receptor; IHD, ischemic heart disease; IL, interleukin; LAAS, large-artery atherosclerosis; LAC, lacunar infarct; LDL, low-density lipoprotein; MMP, matrix metalloproteinase; MRI, magnetic resonance imaging; NIHSS, National Institutes of Health Stroke Scale; ODE, stroke of other determined etiology; PDGF, platelet-derived growth factor; PLT, platelet; PT, prothrombin time; RBC, red blood cell; SBP, systolic blood pressure; SD, standard deviation; TC, total cholesterol; TG, triglyceride; TGF- $\beta$, transforming growth factor- $\beta$; TIMP, tissue inhibitor of metalloproteinase; TNF- $\alpha$, tumor necrosis factor- $\alpha$; TOAST, Trial of ORG10172 in Acute Stroke Treatment; UDE, stroke of undetermined etiology; WBC, white blood cell

Key words: decorin, matrix metalloproteinase-2, stroke, extracellular matrix metalloproteinase-2 (MMP-2) levels and other laboratory and clinical variables. Plasma concentrations of DCN were assessed in 102 patients with AIS (less than 7 days) and 120 control subjects using ELISA assays. The correlation between DCN concentrations and MMP-2 levels, Trial of Org 10172 in Acute Stroke Treatment (TOAST) subtypes, stroke severity and risk factors were evaluated. The expression of DCN was significantly decreased in patients with AIS $(\mathrm{P}<0.001)$, particularly in the large-artery atherosclerosis (LAAS) group. The levels of DCN were positively correlated with MMP-2 $(\mathrm{R}=0.332 ; \mathrm{P}<0.001)$, thus MMP-2 is an independent predictor of DCN concentration $(\mathrm{P}<0.001)$. DCN levels below $8,500 \mathrm{pg} / \mathrm{ml}$ had sensitivity and specificity values of AIS of 79.4 and $62.8 \%$, respectively and DCN below 8,500 pg/ml was associated with AIS (OR=4.8; 95\% CI: 2.1-11.1; $\mathrm{P}<0.001)$ following adjustment for potential confounders. In conclusion, for the first time, a reduction in DCN was detected in patients following AIS and these altered plasma concentrations were correlated with MMP-2. Larger studies are required to further investigate whether DCN is involved in the pathogenesis of ischemic stroke.

\section{Introduction}

Acute ischemic stroke (AIS) is a common cause of morbidity and mortality in industrialized countries. According to the World Health Organization, stroke affects approximately 5.8 million individuals each year worldwide (1). Stroke may trigger an inflammatory reaction that lasts several months. It has been reported that the suppression of inflammation with a variety of drugs reduces infarct volume and improves clinical outcomes in animal models of stroke (2). Extracellular matrix (ECM) remodeling, effected by a group of significant inflammatory molecules, including matrix metalloproteinases (MMPs), may be a key factor in the development of inflammation in the central nervous system. Proteoglycans are thought to cause matrix remodeling and modulate the activity of growth factors and cytokines (3).

Decorin (DCN) is a small proteoglycan that consists of a single glycosaminoglycan side chain and is linked to a core protein containing leucine-rich repeats of 24 amino acids. It is present in the ECM of a variety of tissues and cell types (4). 
During the past decade, accumulating evidence has suggested that DCN interacts with a variety of biological molecules, including type I collagen, fibronectin, thrombospondin, epidermal growth factor receptors (EGF-Rs), insulin-like growth factor-1 receptors (IGF-1Rs), platelet-derived growth factor (PDGF), complement Clq and MMPs, particularly MMP-2 and MMP-9 (5-8). These factors are involved in matrix assembly and may be involved in the regulation of fundamental biological functions, including cell attachment, migration and proliferation (9-12). It has also been reported that DCN is involved in the regulation of collagen fibrillogenesis, ECM remolding or deposition and cancer cell growth. However, no studies have investigated the expression of DCN in patients with AIS and in particular the correlation between DCN and MMP-2 has not been explored.

The present study aimed to investigate the levels of DCN and MMP-2 in normal volunteers as well as in subjects with AIS. Furthermore, the correlations between plasma DCN and MMP-2 and other varying clinical factors in the subjects were analyzed and the role of DCN as a biomarker of risk for AIS was explored.

\section{Subjects and methods}

Subjects and assessment. Patients with AIS were recruited from the Department of Neurology, Changhai Hospital, Second Military Medical University, Shanghai, between April 2010 and May 2011. A total of 120 volunteers matched for age, gender and cardiovascular risk factors, including hypertension, diabetes mellitus (DM), hypercholesterolemia and heart disease, were included as controls. Diagnosis of AIS was defined by focal neurological signs or symptoms of potential vascular origin that persisted for $>24 \mathrm{~h}$ and confirmed using brain computed tomography (CT) and/or magnetic resonance imaging (MRI) within 7 days following the onset of stroke according to The International Classification of Diseases (9th Revision) (13). Exclusion criteria for the two groups were: (a) infectious disease or traumatic injury in the previous month; (b) myocardial infarction in the previous year; (c) severe renal and liver failure; (d) history of any chronic inflammatory disease; (e) history of cancer. Smoking history and history of hypertension, DM, hypercholesterolemia, or any heart disease were recorded.

In this study, stroke subtype was classified according to the Trial of Org 10172 in Acute Stroke Treatment (TOAST) criteria (14): (a) large-artery atherosclerosis (LAAS); (b) cardioembolic infarct (CEI); (c) lacunar infarct (LAC); (d) stroke of other determined etiology (ODE); (e) stroke of undetermined etiology (UDE).

Stroke severity was scored using the National Institutes of Health Stroke Scale (NIHSS) on admission and at $72 \mathrm{~h}$ (15).

This project was performed according to the principles of the Declaration of Helsinki and approved by the local ethics commission. All patients gave informed consent. All data were evaluated by a trained neurologist and a researcher who were blinded to each other's assessment.

Laboratory tests and DCN measurements. The blood samples from all patients were collected by laboratory specialists on the first day of admission. Routine laboratory tests, including red blood cell (RBC), white blood cell (WBC), platelet (PLT), plasma glucose, fibrinogen degradation products (FDP), fibrinogen (FIB), prothrombin time (PT), activated partial thromboplastin time (APTT), triglyceride (TG), total cholesterol (TC), low-density lipoprotein (LDL) and high-density lipoprotein (HDL) were determined using an automatic analyzer (Hitachi 7060, Tokyo, Japan).

Blood samples for DCN and MMP-2 were collected into chilled tubes containing EDTA- $\mathrm{Na}_{2}(1 \mathrm{mg} / \mathrm{ml}$ blood $)$ and centrifuged at $4,000 \mathrm{rpm}$ at $4^{\circ} \mathrm{C}$ for $10 \mathrm{~min}$. The supernatants were decanted and frozen at $-80^{\circ} \mathrm{C}$ until assayed. DCN was measured using enzyme-linked immunosorbent assay (ELISA) with Raybio human DCN ELISA kits (Raybiotech, Norcross, GA, USA) according to the manufacturer's instructions. MMP-2 was treated using R\&D Human MMP-2 Immunoassay kit (R\&D Systems, Minneapolis, MN, USA) following the manual procedure. Intra- and inter-assay coefficients of variation for the two were $<10 \%$.

Statistical analyses. All statistical analyses were performed using SPSS 17.0 software. Values are expressed as mean \pm SD or, in the case of non-normally distributed data, as median and interquartile range. Data that were normally distributed were analyzed using a Student's t-test (for two groups) or one-way analysis of variance (ANOVA; for three groups). Non-normally distributed data were analyzed using the nonparametric Mann-Whitney U test (for two groups) or Kruskal-Wallis test (for three groups). Pearson's correlation coefficient was calculated to evaluate a possible correlation in continuous variables. Multiple stepwise linear regression analyses were performed to identify independent determinants of plasma DCN concentration. Logistic regression analyses were used to assess whether stroke was related to traditional atherosclerotic risk factors and DCN. For the assessment of the accuracy of the parameter of DCN in discriminating between stroke patients and controls, receiver operating characteristic curve (ROC) analyses were performed. Data were considered to be statistically significant when $\mathrm{P}<0.05$.

\section{Results}

Plasma levels of DCN and MMP-2 are decreased in AIS patients. The study population consisted of 102 AIS patients (71 men; 31 women) and 120 controls. Demographic and clinical characteristics are shown in Table I. The vascular risk factors, including ischemic heart disease (IHD), hypertension, atrial fibrillation (AF), DM, hyperlipidemia, smoking and alcohol consumption did not differ significantly between the groups. No significant differences in PLT, PT, APTT, D-dimer, serum triglycerides (TGs), HDL and total cholesterol (TC) were observed between the two groups (Table I).

Plasma levels of DCN were significantly lower in AIS patients compared with individuals in the control group (P<0.001, Table I, Fig. 1). Similarly, plasma levels of MMP-2 were significantly lower in patients than in the controls $(\mathrm{P}<0.001$, Table I, Fig. 1). The plasma DCN levels were positively correlated with MMP-2 $(\mathrm{R}=0.332 ; \mathrm{P}<0.001)$.

Plasma DCN levels are lowest in patients with LAAS. Patients were divided into five subgroups according to the criteria 
Table I. Clinical characteristics and laboratory parameters of patients with and without stroke.

\begin{tabular}{|c|c|c|c|}
\hline Demographic & AIS (n=102) & Control $(n=120)$ & P-value \\
\hline Male, n (\%) & $71(69.6)$ & $76(63.3)$ & 0.393 \\
\hline Age, years & $61.3 \pm 14.4$ & $61.6 \pm 12.2$ & 0.168 \\
\hline \multicolumn{4}{|c|}{ Stroke subtype of etiology (TOAST), n (\%) } \\
\hline Cardioembolic infarcts & 8 & & \\
\hline Large-artery atherosclerosis & 59 & & \\
\hline Lacunar infarct & 8 & & \\
\hline Stroke of other determined etiology & 7 & & \\
\hline Stroke of undetermined etiology & 20 & & \\
\hline \multicolumn{4}{|l|}{ Risk factor, n (\%) } \\
\hline History of ischemic heart disease & $9(8.8)$ & $15(12.5)$ & 0.379 \\
\hline Hypertension & $64(62.7)$ & $77(64.2)$ & 0.274 \\
\hline Diabetes mellitus & $23(22.5)$ & $27(22.5)$ & 0.993 \\
\hline Atrial fibrillation & $10(9.8)$ & $13(10.8)$ & 0.802 \\
\hline Hyperlipidemia & $7(6.9)$ & $10(8.3)$ & 0.681 \\
\hline Cigarette smoking, n (\%) & $56(54.9)$ & $51(42.5)$ & 0.193 \\
\hline Never & $46(45.1)$ & $69(57.5)$ & \\
\hline Former & $23(22.5)$ & $21(17.5)$ & \\
\hline Current & $33(32.4)$ & $30(25)$ & \\
\hline Alcohol intake, n (\%) & $55(53.9)$ & $60(50)$ & 0.801 \\
\hline Non-drinker, n & 47 & 60 & \\
\hline Former-drinker, $\mathrm{n}$ & 34 & 42 & \\
\hline Drinker, $\mathrm{n}$ & 21 & 18 & \\
\hline \multicolumn{4}{|l|}{ Laboratory results } \\
\hline $\mathrm{RBC}\left(\mathrm{x} 10^{12} / \mathrm{l}\right)$ & $4.6 \pm 0.6$ & $4.3 \pm 0.4$ & 0.002 \\
\hline $\mathrm{WBC}\left(\mathrm{x} 10^{9} / \mathrm{l}\right)$ & $7.4 \pm 2.3$ & $6.7 \pm 1.3$ & 0.006 \\
\hline $\operatorname{PLT}\left(\times 10^{9} / 1\right)$ & $206.5 \pm 47.8$ & $202.2 \pm 37.0$ & 0.462 \\
\hline PT (s) & $13.9 \pm 5.0$ & $13.1 \pm 1.6$ & 0.147 \\
\hline APTT (s) & $37.9 \pm 9.4$ & $37.7 \pm 3.8$ & 0.865 \\
\hline $\mathrm{FIB}(\mathrm{g} / \mathrm{l})$ & $3.56 \pm 1.29$ & $3.16 \pm 0.44$ & 0.004 \\
\hline D-dimer $(\mu \mathrm{g} / \mathrm{ml})$ & $1.09 \pm 2.36$ & $0.67 \pm 0.50$ & 0.60 \\
\hline $\mathrm{FDP}(\mu \mathrm{g} / \mathrm{ml})$ & $5.89 \pm 7.41$ & $2.97 \pm 1.31$ & $<0.001$ \\
\hline $\mathrm{LDL}(\mathrm{mmol} / \mathrm{l})$ & $3.08 \pm 1.20$ & $2.56 \pm 0.51$ & $<0.001$ \\
\hline $\operatorname{HDL}(\mathrm{mmol} / \mathrm{l})$ & $1.01 \pm 0.22$ & $1.00 \pm 0.19$ & 0.767 \\
\hline TG (mmol/l) & $1.58 \pm 0.66$ & $1.65 \pm 0.64$ & 0.47 \\
\hline $\mathrm{TC}(\mathrm{mmol} / \mathrm{l})$ & $4.70 \pm 1.17$ & $4.45 \pm 0.74$ & 0.063 \\
\hline Plasma glucose $(\mathrm{mmol} / \mathrm{l})$ & $6.2 \pm 2.1$ & $5.1 \pm 1.4$ & $<0.001$ \\
\hline Decorin (pg/ml) & $7094.8 \pm 2150.67$ & $8950.04 \pm 1267.65$ & $<0.001$ \\
\hline MMP-2 (ng/ml) & $132.37 \pm 37.2$ & $185.92 \pm 33.94$ & $<0.001$ \\
\hline \multicolumn{4}{|l|}{ Vital signs at admission } \\
\hline Body temperature $\left({ }^{\circ} \mathrm{C}\right)$ & $36.5 \pm 0.5$ & $36.6 \pm 0.5$ & 0.078 \\
\hline $\mathrm{SBP}(\mathrm{mmHg})$ & $139.8 \pm 20.0$ & $129.6 \pm 13.4$ & $<0.001$ \\
\hline $\mathrm{DBP}(\mathrm{mmHg})$ & $84.8 \pm 11.0$ & $75.4 \pm 9.5$ & 0.099 \\
\hline Heart rate (/min) & $77.6 \pm 6.5$ & $74.2 \pm 8.9$ & $<0.001$ \\
\hline
\end{tabular}

Continuous variables are expressed as mean \pm SD. APTT, activated partial thromboplastin time; DBP, diastolic blood pressure; FDP, fibrinogen degradation products; FIB, fibrinogen; HDL, high-density lipoprotein; LDL, low-density lipoprotein; PLT, platelet; PT, prothrombin time; RBC, red blood cell; SBP, systolic blood pressure; TC, total cholesterol; TG, triglyceride; TOAST, Trial of ORG10172 in Acute Stroke Treatment; WBC, white blood cell; AIS, acute ischemic stroke.

used in TOAST. The five stroke subgroups were as follows: CEI, n=8; LAAS, n=59; LAC, n=8; ODE, n=7; UDE, $n=20$. The DCN levels were 9183.2 $\pm 1892.65,6640.63 \pm 2050.75$, $7877.87 \pm 2022.54,6966.03 \pm 2104.68$ and $7331.09 \pm 2150.67$ in groups CEI, LAAS, LAC, ODE and UDE, respectively. The lowest DCN levels were identified in patients with LAAS. Patients with CEI demonstrated the highest DCN levels among the five groups. Fig. 3 shows that DCN levels were signifi- 
Table II. Pearson correlation between DCN and clinical laboratory parameters.

\begin{tabular}{lcl}
\hline & \multicolumn{2}{c}{$\mathrm{DCN}$} \\
\cline { 2 - 3 } Patient characteristics & $\mathrm{R}$ & \multicolumn{1}{c}{ P-value } \\
\hline Age & -0.14 & $0.830(\mathrm{NS})$ \\
Systolic blood pressure & -0.188 & $0.005^{\mathrm{b}}$ \\
Diastolic blood pressure & -0.180 & $0.007^{\mathrm{b}}$ \\
Ischemic heart disease & -0.026 & 0.705 (NS) \\
Atril fibrillation & 0.181 & $0.007^{\mathrm{b}}$ \\
Plasma glucose & -0.159 & $0.017^{\mathrm{a}}$ \\
Fibrinogen & -0.045 & 0.509 (NS) \\
FDP & -0.199 & $0.003^{\mathrm{b}}$ \\
D-dimer & -0.122 & 0.069 (NS) \\
Total cholesterol & -0.084 & 0.213 (NS) \\
Triglyceride & 0.059 & $0.382(\mathrm{NS})$ \\
High-density lipoprotein & -0.058 & 0.391 (NS) \\
Low-density lipoprotein & -0.154 & $0.022^{\mathrm{a}}$ \\
NIHSS & 0.12 & $0.906(\mathrm{NS})$ \\
MMP-2 & 0.332 & $<0.001^{\mathrm{b}}$ \\
\hline
\end{tabular}

DCN, decorin; FDP, fibrinogen degradation products; MMP-2, matrix metalloproteinase-2; NS, not significant; NIHSS, National Institutes of Health Stroke Scale. ${ }^{\mathrm{a}} \mathrm{P}<0.05 ;{ }^{\mathrm{b}} \mathrm{P}<0.01$.

Table III. Cut-off values of DCN in AIS and controls.

\begin{tabular}{lcc}
\hline Cut-off $(\mathrm{pg} / \mathrm{ml})$ & Sensitivity $(\%)$ & Specificity $(\%)$ \\
\hline 9,000 & 79.4 & 47.5 \\
8,500 & 73.5 & 60.0 \\
8,000 & 69.6 & 78.3 \\
$8,500^{\mathrm{a}}$ & 79.4 & 62.8 \\
$8,500^{\mathrm{b}}$ & 79.4 & 39.0 \\
\hline
\end{tabular}

${ }^{\mathrm{a} C}$ Controls $(\mathrm{n}=43)$ were individuals without risk factors; ${ }^{\mathrm{b}}$ Controls $(\mathrm{n}=77)$ were individuals with risk factors; DCN, decorin; AIS, acute ischemic stroke.

cantly lower in patients with LAAS, LAC, ODE and UDE than those in controls $(\mathrm{P}<0.001, \mathrm{P}=0.028, \mathrm{P}<0.001, \mathrm{P}<0.001$, respectively). However, no difference was noted in DCN levels in patients with CEI as compared with controls.

DCN levels $<8,500 \mathrm{pg} / \mathrm{ml}$ are associated with AIS. Correlation analyses (Table II) revealed a positive correlation between DCN levels and MMP-2 $(\mathrm{R}=0.332 ; \mathrm{P}<0.001)$ and $\mathrm{AF}(\mathrm{R}=0.181$; $\mathrm{P}=0.007)$. A negative correlation was revealed between DCN levels and systolic blood pressure $(\mathrm{R}=-0.188 ; \mathrm{P}<0.001)$, diastolic blood pressure $(\mathrm{R}=-0.180 ; \mathrm{P}=0.005)$, plasma glucose $(\mathrm{R}=-0.159 ; \mathrm{P}=0.017)$, FDP $(\mathrm{R}=-0.199 ; \mathrm{P}=0.003)$ and $\mathrm{LDL}$ $(\mathrm{R}=-0.154 ; \mathrm{P}=0.022)$. However there was no correlation between DCN levels and NIHSS ( $\mathrm{R}=0.12 ; \mathrm{P}=0.906)$. Multiple stepwise linear regression analyses revealed that MMP-2 and AF were significant independent determinants of DCN
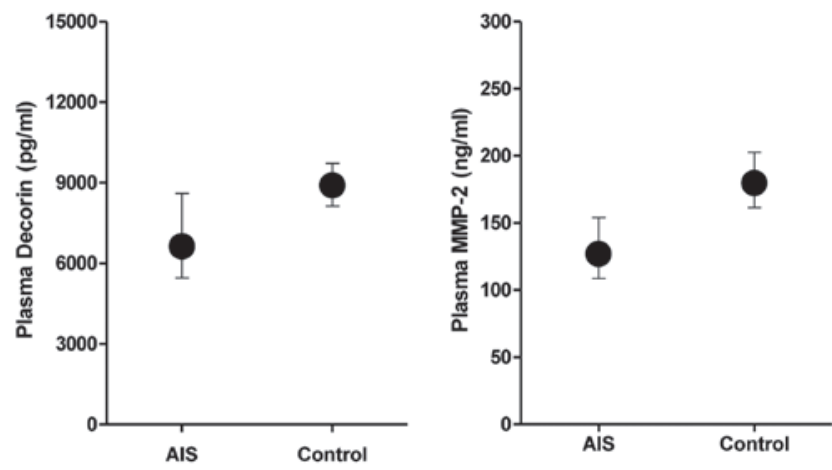

Figure 1. Plasma decorin and MMP-2 levels in subjects with AIS and controls. AIS, acute ischemic stroke.

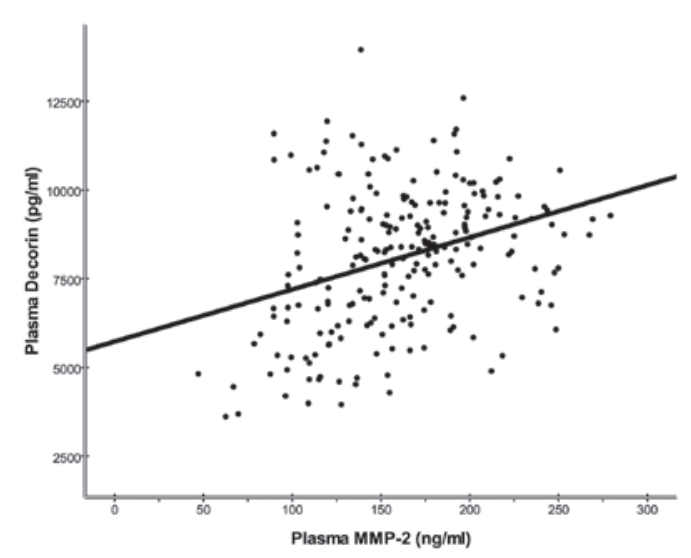

Figure 2. Positive correlation between decorin and MMP-2. MMP-2, matrix metalloproteinase-2.

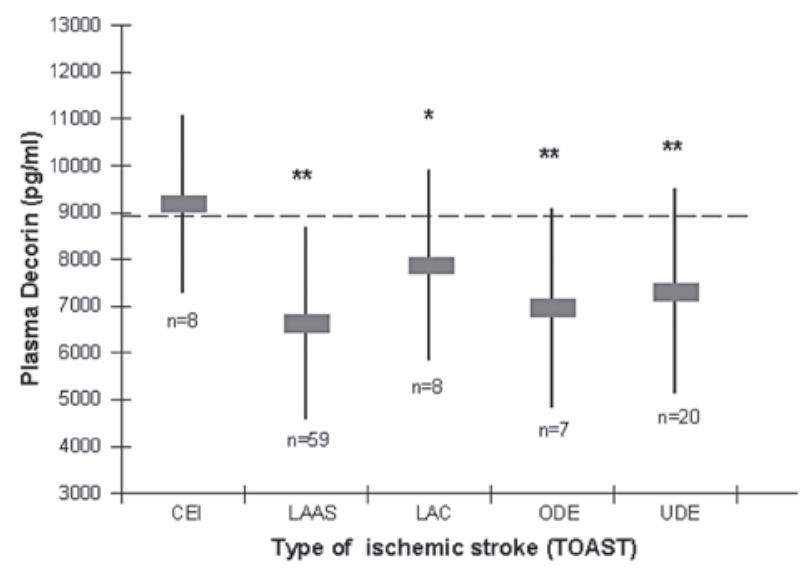

Figure 3. Mean values with 95\% CI of plasma decorin levels in each subgroup of ischemia stroke. The dotted line represents the level of decorin in the control group. CEI, cardioembolic infarct; LAAS, large-artery atherosclerosis; LAC, lacunar infarct; ODE, stroke of other determined etiology; UDE, stroke of undetermned etiology.

( $\beta=0.341, P<0.001 ; \beta=0.197, P=0.002$, respectively), when the vascular risk factors, including age, gender, hypertension, DM, smoking, alcohol, TC, LDL and HDL were taken into account as independent variables. Following adjustment for smoking, alcohol, DM and hypertension, logistic regression analyses revealed that DCN levels $<8,500 \mathrm{pg} / \mathrm{ml}$ were associated with AIS (OR=4.8; 95\% CI: 2.1-11.1; $\mathrm{P}<0.001)$. 

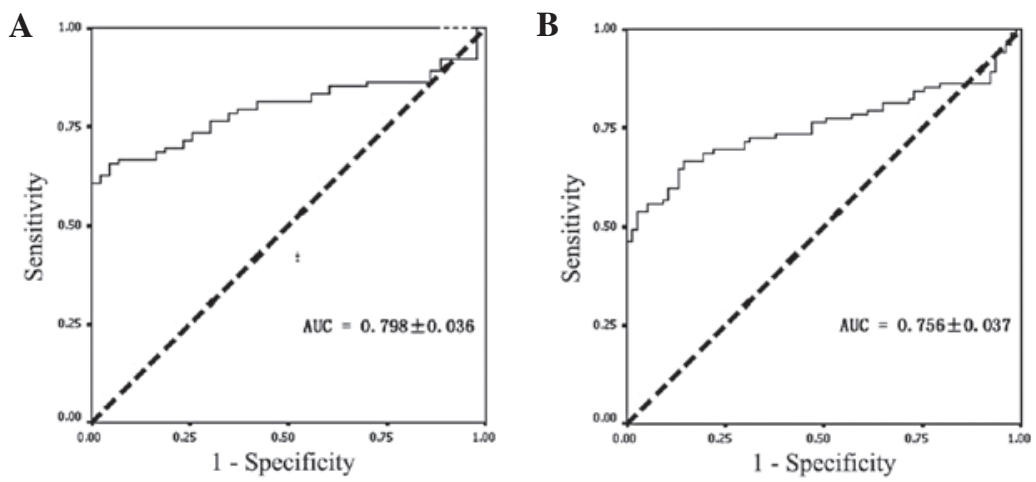

Figure 4. Receiver operating characteristic (ROC) curves for plasma DCN values in subjects with known vascular risk factors (A) and those without (B). AUC, area under the ROC curve; DCN, decorin.

Plasma DCN $(8,500 \mathrm{pg} / \mathrm{ml})$ has a sensitivity and specificity for AIS of 79.4 and $62.8 \%$, respectively. To define the optimal cut-off value for DCN levels, an ROC curve was constructed (Fig. 2). The controls $(\mathrm{n}=120)$ were divided into the patients with known vascular risk factors $(n=77)$ and those without $(n=43)$ and three cut-off values were calculated $(8,000$, 8,500 and $9,000 \mathrm{pg} / \mathrm{ml}$ ) which provided various accuracies (Table III). The cut-off value used for DCN levels was $8,500 \mathrm{pg} / \mathrm{ml}$ and the area under the curve (AUC) for DCN was 0.798 (95\% CI: $0.73-0.87$; $\mathrm{P}<0.001$ ) in the patients with AIS and controls without known vascular risk factors $(n=43)$. The sensitivity and specificity of plasma DCN $(8,500 \mathrm{pg} / \mathrm{ml})$ for AIS were 79.4 and $62.8 \%$, respectively.

\section{Discussion}

DCN is a ubiquitous small extracellular proteoglycan. It is a composite molecule, $100 \mathrm{kDa}$ in size, with a protein core and attached glycosaminoglycans (GAGs) (16). It was cloned from a human embryonic fibroblast line and named PG40 due to its protein core $(40 \mathrm{kDa})$ (4). Previous studies have suggested that DCN is involved in the regulation of collagen fibrillogenesis, ECM remolding, tumor growth and metastasis, angiogenesis, renal and pulmonary fibrosis, muscular dystrophy, wound healing and myocardial infarction (17-20). Although previous studies have furthered our understanding of DCN in various pathological processes, the role of DCN in patients with AIS has not been investigated. The present study examined plasma DCN and MMP-2 in subjects with AIS. For the first time, this study revealed that there are lower plasma levels of DCN in subjects with AIS compared with those in controls. Decreased DCN levels $(<8,500 \mathrm{pg} / \mathrm{ml})$ had $79.4 \%$ sensitivity and $62.8 \%$ specificity for AIS, therefore this cut-off point might be a useful indicator for ischemic stroke.

MMPs underlie a tight regulatory process resulting in the equilibrium between the synthesis and degradation of ECM components (21-23). This equilibrium may be disturbed following stroke, leading to the decreased synthesis of MMP-2. However, conflicting data have reported an increase (24) or decrease (25) in plasma levels following ischemic stroke. The observed decrease in MMP-2 levels following AIS in the current study may be explained pathophysiologically as a failure to detect circulating MMP-2 due to an accelerated MMP turnover, binding of MMP-2 to damaged tissue or increased utilization of MMP-2 for local matrix remodelling (26). In the current study, plasma DCN levels were positively correlated with MMP-2. Therefore, these results are in agreement with those of a previous study, in which a recombinant adenovirus vector containing a DCN gene transfection to rat mesangial and tubular cells led to the repressed expression of transforming growth factor- $\beta$ (TGF- $\beta$ ) and collagen type IV, whilst it promoted the expression of MMP-2 and -9 (27). Similarly, Al Haj Zen et al (28) demonstrated that DCN may affect the production of metalloproteinases and cytokines through the adenovirus-mediated overexpression of DCN in the human gingival fibroblasts. In this study, the authors also demonstrated that DCN infection resulted in the decreased expression of MMP-1, MMP-3, TGF- $\beta$ and IL-1 $\beta$. However, the levels of MMP-2, tissue inhibitor of metalloproteinase -2 (TIMP-2) and IL-4 were markedly increased. Consistent with previous studies, the results of the current study also suggest that plasma DCN levels are involved in the development of ischemic stroke by interacting with MMP-2.

Atherosclerosis, an underlying cause of a large proportion of strokes, is a multifactorial process which synergistically induces oxidative stress and a chronic inflammatory state (29). Lipids, particularly cholesterol transported in circulating LDL particles, are key factors that are associated with the formation of the atherosclerotic plaque. In a previous study, the authors reported a reduction of atherosclerosis development in the animal model of ApoE-/- mice treated using adenoviruses containing the human DCN gene (3). The secreted DCN formed complexes with $40 \%$ of plasma TGF- $\beta 1$ of ApoE-/- mice, leading to a reduction in plasma TGF- $\beta 1$. The overexpression of DCN was accompanied by a reduction in plaque macrophage content. Atherosclerosis protection is achieved since transient $\mathrm{DCN}$ overexpression changes the plaque phenotype to that of lower inflammation. Similarly, in the current study, the level of DCN was the lowest in the LAAS group and it was negatively correlated with LDL-C. This result is consistent with previous studies and suggests that plasma DCN levels are involved in the development of atherosclerosis. The correlation between DCN and NIHSS was also evaluated. NIHSS is a reliable and commonly used score for clinical assessment of the severity of stroke. However, no correlation was identified between DCN levels and NIHSS in the present study. This might be due to the small sample size. Therefore, further studies are required to clarify the correlation between the DCN and NIHSS. 
During AIS, the brain initiates a complex cascade of ischemic events at various levels. Excitotoxicity, oxidative stress, blood-brain barrier dysfunction, inflammation and ECM remolding are the most significant pathophysiological processes involved in this cascade (30-32). Cytokines, including IL-1, IL- 6 , tumor necrosis factor- $\alpha$ (TNF- $\alpha$ ) and TGF- $\beta$ are produced by a variety of activated cell types and are significant mediators of stroke induced inflammation that may contribute to the progression of cerebral infarction (33-35). Yamaguchi et al (36) demonstrated that DCN is a natural inhibitor of transforming growth factor- $\beta 1$ (TGF- $\beta 1$ ). Al Haj Zen et al (3) reported that DCN reduces inflammation by downregulating TGF- $\beta$ and IL-1 $\beta$ expression through adenovirus-mediated overexpression of DCN in the human gingival fibroblasts. These studies indicate that inflammatory cytokines explain the change of DCN expression during stroke.

There are several limitations to the present study. First, the sample size was relatively small. Second, there were no long-term follow-up results. Thus, further studies are required to confirm these preliminary results.

In conclusion, the present study is the first to report that plasma DCN levels are decreased in patients with AIS, particularly in the LAAS group, and that the level of DCN is positively correlated with MMP-2 levels. Of note, decreased plasma DCN concentrations $(<8,500 \mathrm{pg} / \mathrm{ml})$ are associated with increased risk for ischemic stroke. Our findings indicate that DCN is involved in the pathogenesis of ischemic stroke and that a reduction of plasma DCN may be a useful indicator for ischemic stroke.

\section{Acknowledgements}

This study was supported by grants from the Chinese National Science Foundation (no. 30973102). The authors are particularly grateful to the patients who volunteered to participate in this study.

\section{References}

1. Truelsen T, Heuschmann PU, Bonita R, et al: Standard method for developing stroke registers in low-income and middle income countries: experiences from a feasibility study of a stepwise approach to stroke surveillance (STEPS Stroke). Lancet Neurol 6: 134-139, 2007.

2. Saenger AK and Christenson RH: Stroke biomarkers: progress and challenges for diagnosis, prognosis, differentiation and treatment. Clin Chem 56: 21-33, 2010.

3. Al Haj Zen A, Caligiuri G, Sainz J, et al: Decorin overexpression reduces atherosclerosis development in apolipoprotein E-deficient mice. Atherosclerosis 187: 31-39, 2006.

4. Chen S and Birk DE: Focus on molecules: decorin. Exp Eye Res 92: 444-445, 2011.

5. Hu Y, Sun H, Owens RT, et al: Decorin suppresses prostate tumor growth through inhibition of epidermal growth factor and androgen receptor pathways. Neoplasia 11: 1042-1053, 2009.

6. Johnson PY, Potter-Perigo S, Gooden MD, et al: Decorin synthesized by arterial smooth muscle cells is retained in fibrin gels and modulates fibrin contraction. J Cell Biochem 101: 281-94, 2007.

7. Goldoni S, Humphries A, Nyström A, et al: Decorin is a novel antagonistic ligand of the Met receptor. J Cell Biol 185: 743-754, 2009.

8. Reed CC, Waterhouse A, Kirby S, et al: Decorin prevents metastatic spreading of breast cancer. Oncogene 24: 1104-1110, 2005.

9. Iozzo RV, Moscatello DK, McQuillan DJ and Eichstetter I: Decorin is a biological ligand for the epidermal growth factor receptor. J Biol Chem 274: 4489-4492, 1999.

10. Schönherr E, Sunderkotter C, Iozzo RV and Schaefer L: Decorin, a novel player in the insulin-like growth factor system. J Biol Chem 280: 15767-15772, 2005.
11. Kresse H and Schönherr E: Proteoglycans of the extracellular matrix and growth control. J Cell Physiol 189: 266-274, 2001.

12. Merle B, Durussel L, Delmas PD and Clezardin P: Decorin inhibits cell migration through a process requiring its glycosaminoglycan side chain. J Cell Biochem 75: 538-546, 1999.

13. Chen J, Yu H, Sun K, et al: Promoter variant of angiopoietin-2 and plasma angiopoietin-2 are associated with risk of stroke recurrence in lacunar infarct patients. Biochem Biophys Res Commun 398: 212-216, 2010.

14. Adams HP Jr, Bendixen BH, Kappelle LJ, et al: Classification of subtype of acute ischemic stroke. Definitions for use in a multicenter clinical trial. TOAST. Trial of Org 10172 in Acute Stroke Treatment. Stroke 24: 35-41, 1993.

15. Brott T, Adams HP Jr, Olinger CP, et al: Measurements of acute cerebral infarction: a clinical examination scale. Stroke 20: 864-870, 1989.

16. Yan W, Wang $\mathrm{P}$, Zhao CX, et al: Decorin gene delivery inhibits cardiac fibrosis in spontaneously hypertensive rats by modulation of transforming growth factor- $\beta /$ Smad and $\mathrm{p} 38$ mitogen-activated protein kinase signaling pathways. Hum Gene Ther 20: 1190-1200, 2009

17. Zhang Z, Garron TM, Li XJ, et al: Recombinant human decorin inhibits TGF- $\beta 1$-induced contraction of collagen lattice by hypertrophic scar fibroblasts. Burns 35: 527-537, 2009.

18. Zheng F, Lu W, Wu F, et al: Recombinant decorin ameliorates the pulmonary structure alterations by down-regulating transforming growth factor- $\beta 1 /$ SMADS signaling in the diabetic rats. Endocr Res 35: 35-49, 2010.

19. Sanches JC, Jones CJ, Aplin JD, et al: Collagen fibril organization in the pregnant endometrium of decorin-deficient mice. $J$ Anat 216: 144-155, 2010.

20. Schaefer L and Iozzo RV: Biological functions of the small leucine-rich proteoglycans: from genetics to signal transduction. J Biol Chem 283: 21305-21309, 2008.

21. Murphy G: Matrix metalloproteinases and their inhibitors. Acta Orthop Scand Suppl 266: 55-60, 1995.

22. de Nooijer R, Verkleij CJ, von der Thüsen JH, et al: Lesional overexpression of matrix metallopmteinase- 9 promotes intraplaque hemorrhage in advanced lesions but not at earlier stages of atherogenesis. Arterioscler Thromb Vasc Biol 26: 340-346, 2006.

23. Bäck M, Ketelhuth DF and Agewall S: Matrix metalloproteinases in atherothrombosis. Prog Cardiovasc Dis 52: 410-428, 2010.

24. Horstmann S, Kalb P, Koziol J, et al: Profiles of matrix metalloproteinases, their inhibitors and laminin in stroke patients: influence of different therapies. Stroke 34: 2165-2170, 2003.

25. Vukasovic I, Tesija-Kuna A, Topic E, et al: Matrix metalloproteinases and their inhibitors in different acute stroke subtypes. Clin Chem Lab Med 44: 428-434, 2006.

26. Horstmann S, Su Y, Koziol J, et al: MMP-2 and MMP-9 levels in peripheral blood after subarachnoid hemorrhage. J Neurol Sci 251: 82-6, 2006.

27. Dong FQ, Li H, Wu F, et al: Effects of overexpression of decorin on matrix metalloproteinases 2 and 9 in rat mesangial and tubular cells. Zhonghua Yi Xue Za Zhi 88: 3444-3447, 2008 (In Chinese).

28. Al Haj Zen A, Lafont A, Durand E, et al: Effect of adenovirusmediated overexpression of decorin on metalloproteinases, tissue inhibitors of metalloproteinases and cytokines secretion by human gingival fibroblasts. Matrix Biol 22: 251-258, 2003.

29. Ross R: Atherosclerosis is an inflammatory disease. Am Heart J 138: 419-420, 1999.

30. Nilupul Perera M, Ma HK, Arakawa S, et al: Inflammation following stroke. J Clin Neurosci 13: 1-8, 2006.

31. Elkind MS: Inflammatory mechanisms of stroke. Stroke 41 (Suppl 10): S3-8, 2010.

32. Tuttolomondo A, Di Sciacca R, Di Raimondo D, et al: Inflammation as a therapeutic target in acute ischemic stroke treatment. Curr Top Med Chem 9: 1240-1260, 2009.

33. Tuttolomondo A, Di Raimondo D, Di Sciacca R, et al: Fetuin-A and CD40 L plasma levels in acute ischemic stroke: Differences in relation to TOAST subtype and correlation with clinical and laboratory variables. Atherosclerosis 208: 290-296, 2010.

34. Kiewert C, Mdzinarishvili A, Hartmann J, et al: Metabolic and transmitter changes in core and penumbra after middle cerebral artery occlusion in mice. Brain Res 1312: 101-107, 2010.

35. Huang J, Upadhyay UM and Tamargo RJ: Inflammation in stroke and focal cerebral ischemia. Surg Neurol 66: 232-245, 2006.

36. Yamaguchi Y, Mann DM and Ruoslahti E: Negative regulation of transforming growth factor- $\beta$ by the proteoglycan decorin. Nature 346: 281-4, 1990. 